\title{
ÍNDICE DE VULNERABILIDAD DEL RECURSO HÍDRICO CON FINES DE RIEGO EN LA CUENCA HIDROGRÁFICA DEL RÍO NARANJO, CUBA
}

\section{INDEX OF VULNERABILITY OF WATER RESOURCES FOR IRRIGATION IN THE BASIN OF NARANJO RIVER, CUBA}

\author{
Yoandris García-Hidalgo \\ Universidad de Las Tunas, Cuba
}

\begin{abstract}
RESUMEN
El análisis de vulnerabilidad de la cuenca del río Naranjo, es un estudio técnico de diagnóstico que aporta información básica y estratégica para el plan de gestión integral del recurso hídrico. Para el estudio, se tomaron seis puntos representativos de la cuenca. La vulnerabilidad de la cuenca se determinó a través de los indicadores del régimen hidrológico y calidad del agua en base a los criterios de FAO para aguas de riego. Come resultado de determinó que el índice de la vulnerabilidad por desabastecimiento se ha incrementado de moderada a alta, es atribuido por la muy baja capacidad de regulación y retención hídrica y el uso moderado del recurso. Las aguas son no aptas para el riego de los cultivos agrícolas, tienen restricciones ligeras a moderadas. Existe peligro de alcalinización de los suelos producto de las concentraciones de bicarbonato en las aguas. La vulnerabilidad podría incrementarse con la presencia de algunos factores derivados con el cambio climático, por efecto de reducción en la oferta y por una mayor demanda de agua de las actividades agrícolas de la zona.
\end{abstract}

Palabras clave: vulnerabilidad, aptitud para el riego, retención hídrica, stress hídrico cuenca río Naranjo

1 Doctor en Ciencias Técnicas Agropecuarias, Profesor Auxiliar de la Facultad de Agronomía, Universidad de Las Tunas, provincia Las Tunas, Cuba. Correo electrónico: yoandrisgh@ult.edu.cu

Fecha de recepción: 18 de agosto de 2016

Fecha de aceptación: 14 de octubre de 2016 
Yoandris Garcia-Hidalgo. Index of vulnerability of water resources for irrigation in the basin of Naranjo river, Cuba

\begin{abstract}
The vulnerability analysis of the Naranjo River Basin is a technical diagnostic study that provides basic and strategic information for the comprehensive management plan for water resources. For this study, six representative sections of the basin were considered. The vulnerability of the basin was determined through indicators of the hydrological regime and water quality based on FAO criteria for irrigation water. As a result, it was determined that the vulnerability index due to shortages has increased from moderate to high, and it has been attributed to the very low capacity for regulation and water retention and moderate use of the resource. The waters are not suitable for irrigation of agricultural crops, and they have slight to moderate restrictions. There is a danger of soil alkalization resulting from concentrations of bicarbonate in the water. The vulnerability could increase with the presence of some factors derived from climate change, due to the reduced supply and increased demand for water for agricultural activities in the area.
\end{abstract}

Keywords: vulnerability, suitability for irrigation, water retention, water stress Naranjo River Basin

\title{
Introducción
}

El agua es un elemento fundamental para la vida de los seres humanos, está presente en todas las actividades que realizan y hasta hoy no se conoce otra sustancia que pueda sustituirla, por esa razón, se considera que es el recurso que definirá el desarrollo sostenible. (Troballes, 2015).

Hay evidencia que los recursos hídricos son vulnerables al cambio climático y que las consecuencias sobre la sociedad y los ecosistemas dependen de las medidas de adaptación (IPCC, 2007; 2008).

El análisis de vulnerabilidad en las cuencas hidrográficas, es un estudio técnico de diagnóstico que aporta información básica y estratégica para el plan de gestión integral del recurso hídrico. La vulnerabilidad de dichos sistemas se determina a través de los indicadores del régimen hidrológico y calidad del agua en base a los criterios de FAO para aguas de riego.

El estrés hídrico y los problemas de calidad del agua son los eventos adversos más probables a escala global (IPCC, 2008). Se estima además, una mayor demanda de agua para el riego en los climas cálidos, lo cual puede generar un incremento de la competencia por el uso entre los distintos sectores (Magrin et.al., 2007; Rosenzweig et.al, 2004).

La calidad de las aguas, junto a la cantidad, es uno de los indicadores del componente sistemas ambientales para calcular el índice de sostenibilidad ambiental que considera la habilidad de los países para proteger el medio ambiente en las próximas décadas (Chaves y Alipaz, 2007). Esos indicadores son identificados por las Naciones Unidas para definir el desarrollo sostenible. De ahí la importancia de su estudio en las cuencas hidrográficas. 
Es necesario, por tanto, analizar la vulnerabilidad de los recursos hídricos, para cualificar el grado de fragilidad de la fuente con respecto al abastecimiento y a la amenaza de sequía cuando se presentan períodos con condiciones climáticas extremas (IPCC, 2008; Sharma, 2009). La vulnerabilidad se puede abordar evaluando la susceptibilidad de los sistemas hídricos para conservar y mantener su régimen hidrológico actual y determinando la vulnerabilidad de los sectores usuarios del recurso, ante la amenaza de cambios sustanciales en el régimen hidrológico (Salazar, 2008).

Referentes de estudios en esta temática se pueden citar varios ejemplo de ellos son los estudios: Vulnerabilidad de los recursos hídricos de México ante el cambio climático (2015), en colaboración con la CONAGUA, ha impulsado los estudios de vulnerabilidad de los recursos hídricos en México. En la actualidad los pronósticos mundiales dicen que el planeta está sufriendo un calentamiento y que este calentamiento debiera irse acelerando en las próximas décadas, lo que pondrá en juego toda la capacidad de la humanidad para adaptarse a una condición climática bastante distinta a la actual.

Esta investigación tiene como objetivo realizar una evaluación de la vulnerabilidad del recurso hídrico con fines de riego en la cuenca hidrográfica del río Naranjo.

\section{Materiales y métodos}

La zona de estudio se localiza entre las coordenadas: $20^{\circ} 39^{\prime} 00^{\prime \prime}-21^{\circ}$ 01' 00" latitud norte y $76^{\circ} 36^{\prime} 30^{\prime \prime}-76^{\circ} 52^{\prime} 30^{\prime \prime}$ longitud oeste, en el municipio Majibacoa, provincia Las Tunas, Cuba. Figura I. Su superficie es de $412,8 \mathrm{~km}^{2}$, por lo que representa el 7,8 \% de la cuenca del río Cauto de la cual es tributaria. 
Yoandris Garcia-Hidalgo. Index of vulnerability of water resources for irrigation in the basin of Naranjo river, Cuba

Figura I. Cuenca hidrográfica del río Naranjo, Las Tunas, Cuba.

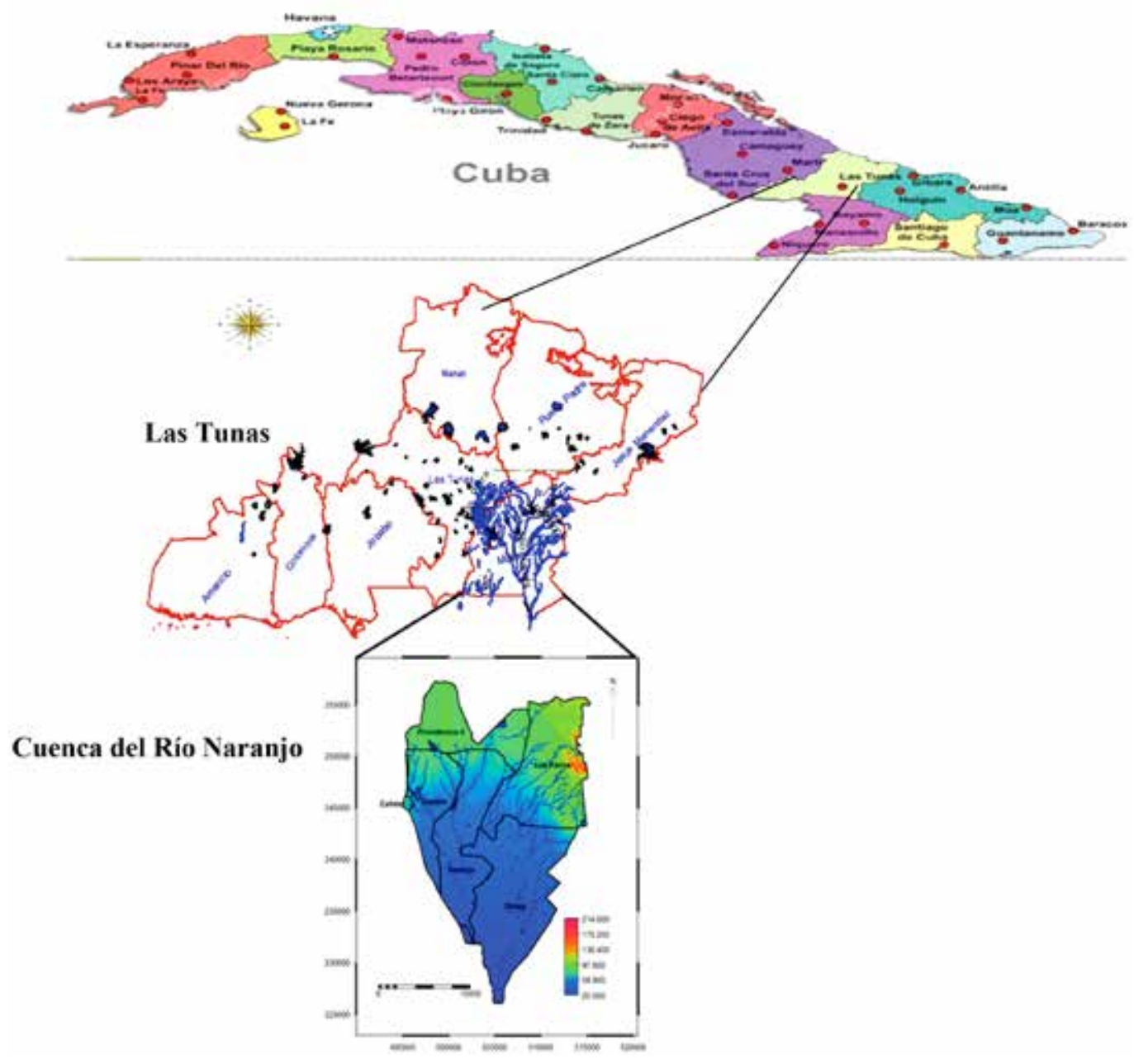

La vulnerabilidad del recurso hídrico fue analizada considerando la susceptibilidad de la cuenca para conservar y mantener el régimen hidrológico actual ante posibles alteraciones por variabilidad climática o por la acción antrópica. Se emplearon los indicadores del régimen hidrológico, sugeridos por el IDEAM, (2010).

Se tomó referente metodológico los sistemas de indicadores para la evaluación de riesgos propuestos por Carreño, (2013). 


\section{Indicadores evaluados}

Los indicadores evaluados fueron: índice de retención y regulación hídrica, índice de aridez, el índice de stress hídrico relativo y los indicadores de calidad del agua según las directrices para interpretar la calidad de las aguas para riego definidos por Ayres y Westcot (1987). La metodología para el cálculo de cada uno de los índices se describe a continuación:

Índice de Retención y Regulación Hídrica (IRH): mide la capacidad de la cuenca para mantener un régimen de caudales; la interpretación se presenta en la Tabla I. Se calcula a partir de la curva de duración de caudales medios diarios, según la ecuación (IDEAM, 2010):

$$
I R H=V P / V_{t} \quad \mathrm{EC}: 1
$$

VP: Volumen representado por el área que se encuentra por debajo de la línea del caudal medio

$\mathrm{V} t$ : Volumen total representado por el área bajo la curva.

Tabla I: Calificación del Índice de Retención y Regulación Hídrica (IRH)

\begin{tabular}{|c|c|l|}
\hline IRH & Calificación cualitativa & \multicolumn{1}{|c|}{ Descripción } \\
\hline$>0,85$ & Muy alta & Muy Alta retención y regulación de Humedad \\
\hline $0,75-0,85$ & Alta & Alta retención y regulación de humedad \\
\hline $0,65-0,75$ & Moderada & Media retención y regulación de humedad \\
\hline $0,50-0,65$ & Baja & Baja retención y regulación de humedad \\
\hline$<0,5$ & Miry Baja & Muy baja retención y regulación de humedad \\
\hline
\end{tabular}

Fuente: IDEAM (2010).

Índice de Aridez: representa la dinámica superficial del suelo y evidencia los lugares con déficit o excedentes de agua; es determinado a partir de la evapotranspiración potencial y real, empleando la ecuación (2); la interpretación se presenta en la Tabla II (IDEAM, 2010):

$$
I_{a}=\frac{E T P-E T R}{E T P} \quad \mathrm{EC}: 2
$$


Yoandris Garcia-Hidalgo. Index of vulnerability of water resources for irrigation in the basin of Naranjo river, Cuba

Donde:

$\mathrm{I}_{a:}$ Índice de aridez

ETP: Evaporación potencial (mm)

ETR: Evaporación real (mm)

Tabla II: Calificación del Índice de aridez

\begin{tabular}{|c|c|l|}
\hline Índice de Aridez & Calificación cualitativa & \multicolumn{1}{|c|}{ Descripción } \\
\hline$<0,15$ & Altos excedentes & Altos excedentes de agua \\
\hline $0,15-0,19$ & Excedentes & Excedentes de agua \\
\hline $0,20-0,29$ & Moderado y excedentes & Moderado y excedentes de agua \\
\hline $0,30-0,39$ & Moderado & Moderado \\
\hline $0,40-0,49$ & Bajo & Moderado y deficitario de agua \\
\hline $0,50-0,59$ & Deficitario & Deficitario de agua \\
\hline$>0,60$ & Altamente deficitario & Altamente deficitario de agua \\
\hline
\end{tabular}

Fuente: IDEAM (2010).

Índice de stress hídrico relativo: El programa mundial de Evaluación de los Recursos Hídricos de la UNESCO, ha definido una serie de indicadores para medir el nivel de estrés sobre el recurso que incluye el índice de stress hídrico relativo también conocido como demanda de agua relativa (RWSI)- Tabla III, establece la demanda disponible para uso industrial, doméstico y de agricultura. Se calcula a partir de la siguiente expresión (UNESCO, 2009):

$$
R W S I=\frac{D+1+A}{Q} \quad \mathrm{EC}: 3
$$

Donde:

Q: suministro de agua- $\mathrm{km}^{3} /$ año

D: Demanda de agua para uso doméstico- $\mathrm{km}^{3} / \mathrm{año}$

I: Demanda de agua para uso industrial- $\mathrm{km}^{3} /$ año

A: Demanda de agua para la agricultura $-\mathrm{km}^{3} / \mathrm{año}$ 
Tabla III: Umbrales del índice de stress hídrico

\begin{tabular}{|c|c|l|}
\hline Índice de Escasez & $\begin{array}{c}\text { Calificación } \\
\text { cualitativa }\end{array}$ & \multicolumn{1}{|c|}{ Observaciones } \\
\hline$>0,4$ & Alto & $\begin{array}{l}\text { Existe fuerte presión sobre el recurso hídrico. Es insuficiente la } \\
\text { oferta hídrica para atender la alta demanda de agua por los } \\
\text { sectores productivos y se restringe el desarrollo económico. Se } \\
\text { requieren fuertes inversiones para mejorar la eficiencia de } \\
\text { utilización en los sectores productivos }\end{array}$ \\
\hline $0,2-0,4$ & Medio & $\begin{array}{l}\text { La oferta hidrica llega al límite máximo para atender la } \\
\text { demanda. Es necesario el ordenamiento de la cuenca y asignar } \\
\text { prioridades a los distintos usos. }\end{array}$ \\
\hline $0,10-0,2$ & Moderado & $\begin{array}{l}\text { La disponibilidad de agua se puede convertir en factor limitante } \\
\text { del desarrollo. Se debe implementar un mejor sistema de } \\
\text { monitoreoy seguimiento a largo plazo }\end{array}$ \\
\hline$<0,1$ & Bajo & $\begin{array}{l}\text { No se experimental presiones sobre el recurso hidrico en } \\
\text { términos de cantidad de agua }\end{array}$ \\
\hline
\end{tabular}

(UNESCO, 2009; IDEAM, 2008)

Fuente: IDEAM (2010).

\section{Indicadores de calidad de las aguas para el riego}

Para determinar la aptitud del agua para el riego se utilizaron los siguientes criterios:

1. Diagrama de Wilcox: para determinar la clasificación de las aguas de acuerdo a sus contenidos de sales solubles totales y de sodio.

2. Salinidad efectiva (SE) ${ }^{2}$ :

a) $\mathrm{Si} \mathrm{el} \mathrm{Ca}{ }^{2+}>\mathrm{CO}_{3}{ }^{2-}+\mathrm{H} \mathrm{CO}_{3}{ }^{-}+\mathrm{SO}_{4}{ }^{2-}$ entonces:

$$
\mathrm{SE}=\sum\left(\mathrm{CO}_{3}^{2-}+\mathrm{HCO}_{3}^{-}+\mathrm{SO}_{4}^{2-}\right)
$$

b) $\mathrm{Si} \mathrm{el} \mathrm{Ca}^{2+}<\mathrm{CO}_{3}^{2-}+\mathrm{HCO}_{3}^{-}+\mathrm{SO}_{4}^{2-}$ pero $\mathrm{Ca}^{2+}>\left(\mathrm{CO}_{3}^{2-}+\mathrm{HCO}_{3}^{-}\right)$ entonces:

$$
S E=\sum \mathrm{Ca}^{2+}
$$

c) $\mathrm{SielCa}{ }^{2+}<\mathrm{CO}_{3}{ }^{2-}+\mathrm{HCO}_{3}{ }^{-}$pero $\mathrm{Ca}^{2+}+\mathrm{Mg}^{2+}>\mathrm{CO}_{3}{ }^{2-}+\mathrm{HCO}_{3}^{-}$entonces:

$$
\mathrm{SE}=\sum\left(\mathrm{CO}_{3}^{2-}+\mathrm{HCO}_{3}^{-}\right)
$$

d) $\mathrm{Si}$ el $\mathrm{Ca}^{2+}+\mathrm{Mg}^{2+}<\mathrm{CO}_{3}^{2-}+\mathrm{HCO}_{3}^{-}$entonces:

$$
S E=\sum\left(\mathrm{Ca}^{2+}+\mathrm{Mg}^{2+}\right)
$$

2 En todos los casos las concentraciones de los iones se expresan en me.1-1. 
3. Salinidad potencial (SP):

$$
\mathrm{SP}=\mathrm{Cl}^{-}+\frac{\mathrm{SO}_{4}^{2-}}{2}
$$

4. Relación de Adsorción de Sodio corregida (SARx):

$$
S A R x=\frac{N a^{+}}{\sqrt{\frac{C a x^{2+}+M g^{2+}}{2}}}
$$

5. Porciento de Sodio Posible (PSP):

$$
P S P=\frac{N a^{+}}{S E} * 100
$$

6. Coeficiente de irrigación $(\mathrm{Ka})$ :

a) $\quad \mathrm{Na}<\mathrm{Cl}^{-}$; el coeficiente viene dado por la expresión: $\mathrm{Ka}=\frac{288}{\mathrm{SCl}}$

b) $\mathrm{Cl}^{-}+\mathrm{SO}_{4}{ }^{2-}>\mathrm{Na}^{+}>\mathrm{Cl}^{-}$el coeficiente vendrá expresado por:

$$
\mathrm{Ka}=\frac{288}{\mathrm{Na}^{+}+4 \mathrm{Cl}^{-}}
$$

c) $\mathrm{rNa}^{+}>\mathrm{rCl}^{-}+\mathrm{rSO}_{4}^{2-}$ el coeficiente viene dado por:

$$
\mathrm{Ka}=\frac{288}{10 \mathrm{Na}^{+}-\mathrm{SCl}^{-}+9 \mathrm{SO}_{4}^{2-}}
$$

7. Índice de saturación de Langelier (IL):

$$
I L=p H_{\text {real }}-p H_{c}
$$

$\mathrm{pH}_{\mathrm{c}}$ : es un valor teórico calculado del $\mathrm{pH}$ del agua de riego en contacto con calcio y en equilibrio con el $\mathrm{CO}_{2}$ de suelo. Se halla en función de los cationes calcio, magnesio y sodio, y los aniones carbonato y bicarbonato (Fuentes, 2003).

\section{Resultados y discusión}

\section{Análisis de Vulnerabilidad}

La vulnerabilidad del recurso hídrico fue evaluada considerando la susceptibilidad de la cuenca para conservar y mantener el régimen 
hidrológico. Para interpretar y analizar los procesos hidrológicos y estimar la vulnerabilidad de la cuenca, se emplean indicadores que permiten caracterizar el régimen hidrológico para el período 1990-2013.

\section{Índice de Retención y Regulación Hídrica (IRH)}

El alto coeficiente de variación de las series de caudal que fluctúa desde el 59\% en la Red Cal 419, hasta el $91 \%$ en la Red Cal 429, se demuestra la baja capacidad de regulación hídrica de la fuente. E1 IRH calculado con los caudales simulados (Q Simulados) es ligeramente mayor que el obtenido con los caudales observados (Q Observados).

Tabla IV: Índice de retención y regulación hídrica- IRH. 1990-2013.

\begin{tabular}{|c|c|c|c|c|c|c|c|}
\hline \multirow{2}{*}{$\begin{array}{l}\text { RED } \\
\text { CAL }\end{array}$} & \multicolumn{3}{|c|}{ IRH (Q Observados) } & \multicolumn{3}{|c|}{ IRH (Q Simulados) } & \multirow{2}{*}{$\begin{array}{l}\text { Categoría } \\
\text { IRH }<0,5\end{array}$} \\
\hline & $\begin{array}{c}\text { RH } \\
\text { promedio }\end{array}$ & IRHp50 & $\begin{array}{l}\text { IRH } \\
\text { INT }\end{array}$ & $\begin{array}{c}\text { IRH } \\
\text { promedio }\end{array}$ & IRHp50 & IRHINT. & \\
\hline 419 & 0,34 & 0,25 & $\mathbf{0 , 3 1}$ & 0,41 & $\mathbf{0 , 3 0}$ & 0,36 & Muy bajo \\
\hline 420 & 0,36 & 0,22 & 0,34 & 0,35 & 0,23 & $\mathbf{0 , 3 2}$ & Muy bajo \\
\hline 424 & $\mathbf{0 , 4 3}$ & $\mathbf{0 , 3 2}$ & $\mathbf{0 , 3 8}$ & 0,42 & $\mathbf{0 , 3 0}$ & $\mathbf{0 , 3 7}$ & Muy bajo \\
\hline 425 & $\mathbf{0 , 3 7}$ & 0,26 & $\mathbf{0 , 3 3}$ & 0,42 & $\mathbf{0 , 3 1}$ & $\mathbf{0 , 3 7}$ & Muy bajo \\
\hline 429 & $\mathbf{0 , 3 1}$ & 0,24 & 0,41 & 0,45 & $\mathbf{0 , 3 0}$ & $\mathbf{0 , 3 8}$ & Muy bajo \\
\hline
\end{tabular}

Fuente: Elaboración propia (2016).

\section{Índice de Aridez}

Para el período 1990-2013, los valores medios del índice de aridez, determinados a partir de la evapotranspiración potencial y real se presentan en la Tabla V. En las secciones media-alta y baja de la cuenca se tienen excedentes de aguas; mientras que en secciones altas y media son moderados y con excedentes de agua. El índice de aridez varía de 0,20 a 0,18.

Tabla V. Índice de Aridez 1990-2013

\begin{tabular}{|c|c|c|c|}
\hline \multirow{2}{*}{ RED - CAL } & \multicolumn{3}{|c|}{ Índice de Aridez } \\
\cline { 2 - 4 } & Valor promedio & CV & Categoría \\
\hline 419 & $\mathbf{0 , 1 8}$ & $\mathbf{3 7 \%}$ & Excedentes \\
\hline 420 & $\mathbf{0 , 1 9}$ & $\mathbf{3 6 \%}$ & Excedentes \\
\hline 424 & $\mathbf{0 , 1 9}$ & $\mathbf{3 5 \%}$ & Excedentes \\
\hline 425 & $\mathbf{0 , 2 0}$ & $\mathbf{3 5 \%}$ & Moderado y excedentes \\
\hline 429 & $\mathbf{0 , 2 0}$ & $\mathbf{3 5 \%}$ & Moderado y excedentes \\
\hline
\end{tabular}

Fuente: Elaboración propia (2016). 


\section{Índice de stress hídrico relativo}

Existe fuerte presión sobre el recurso hídrico en tres de la redes en estudio. Estas zonas de producción de cultivos varios son exigentes de alta demanda de agua, demostrando estos resultados la insuficiente oferta hídrica para atender la alta demanda de agua para estos sectores productivos. Este indicador ha influido en gran medida en la reducción del crecimiento vegetativo y por la duración y el momento de aplicación del riego, siendo más sensible durante la fase de crecimiento activo.

Las categorías medio, se identifican en dos zonas. La oferta hídrica llega al límite máximo para atender la demanda. Es necesario el ordenamiento de la cuenca y asignar prioridades a los distintos usos en especial las aguas con fines agrícolas.

Tabla VI. Índice de stress hídrico relativo.

\begin{tabular}{|c|c|c|}
\hline RED - CAL & Índice de Escasez & Categoría \\
\hline 419 & 0.5 & Alto \\
\hline 420 & 0.6 & Alto \\
\hline RED - CAL & Índice de Escasez & Categoría \\
\hline 424 & 0.4 & Medio \\
\hline 425 & 0.3 & Medio \\
\hline 429 & 0.5 & Alto \\
\hline
\end{tabular}

Fuente: Elaboración propia (2016).

\section{Indicadores de calidad del agua (Criterios generales de la FAO)}

En la Tabla VII se puede apreciar la composición físico-química de las aguas de las fuentes estudiadas en la cuenca del río Naranjo. Las filas Húmedo y Seco identifican las fechas de muestreo. 
Tabla VII. Composición físico-química de las aguas para uso agrícola en la cuenca del río Naranjo.

\begin{tabular}{|c|c|c|c|c|c|c|c|c|}
\hline \multirow{2}{*}{ Variables } & \multirow{2}{*}{ Unidades } & \multirow{2}{*}{ Período } & \multicolumn{6}{|c|}{ Muestras } \\
\hline & & & I & II & III & IV & $\mathbf{V}$ & VI \\
\hline \multirow[t]{2}{*}{$\overline{\mathrm{pH}}$} & \multirow[t]{2}{*}{$\mathrm{U}$} & Húmedo & 7,52 & 7,54 & 7,85 & 8,03 & 7,99 & 7,32 \\
\hline & & Seco & 8,11 & 8,25 & 8,33 & 8,32 & 8,05 & 7,79 \\
\hline \multirow{2}{*}{$\begin{array}{l}\text { Conductividad } \\
\text { Eléctrica }\end{array}$} & \multirow[t]{2}{*}{$\mathrm{dS} / \mathrm{m}^{-1}$} & Húmedo & 2,96 & 2,98 & 0,71 & 0,92 & 0,92 & 5,51 \\
\hline & & Seco & 3,81 & 2,96 & 1,35 & 1,15 & 1,14 & 1,84 \\
\hline \multirow[t]{2}{*}{ Calcio } & \multirow[t]{2}{*}{ me. $^{-1}$} & Húmedo & 5,80 & 5,90 & 1,85 & 1,75 & 1,75 & 28,60 \\
\hline & & Seco & 4,45 & 7,05 & 2,00 & 2,30 & 2,00 & 3,65 \\
\hline \multirow[t]{2}{*}{ Magnesio } & \multirow[t]{2}{*}{ me..$^{-1}$} & Húmedo & 8,43 & 8,35 & 0,91 & 1,82 & 1,82 & 19,09 \\
\hline & & Seco & 4,38 & 8,51 & 1,49 & 1,57 & 1,32 & 6,03 \\
\hline \multirow[t]{2}{*}{ Sodio } & \multirow[t]{2}{*}{ me..$^{-1}$} & Húmedo & 18,09 & 18,61 & 4,39 & 5,35 & 5,35 & 10,17 \\
\hline & & Seco & 32,26 & 12,43 & 10,22 & 8,57 & 11,87 & 8,57 \\
\hline \multirow[t]{2}{*}{ Potasio } & \multirow[t]{2}{*}{ me..$^{-1}$} & Húmedo & 0,03 & 0,03 & 0,20 & 0,13 & 0,13 & 0,15 \\
\hline & & Seco & 0,03 & 0,10 & 0,10 & 0,15 & 0,10 & 0,05 \\
\hline \multirow[t]{2}{*}{ Carbonatos } & \multirow[t]{2}{*}{ me..$^{-1}$} & Húmedo & 0,00 & 0,00 & 0,00 & 0,00 & 0,00 & 0,00 \\
\hline & & Seco & 0,00 & 0,00 & 0,00 & 0,00 & 0,00 & 0,00 \\
\hline \multirow[t]{2}{*}{ Bicarbonato } & \multirow[t]{2}{*}{ me..$^{-1}$} & Húmedo & 12,38 & 12,43 & 4,59 & 4,30 & 4,30 & 4,87 \\
\hline & & Seco & 16,18 & 8,46 & 8,30 & 5,21 & 8,43 & 6,82 \\
\hline \multirow[t]{2}{*}{ Sulfato } & \multirow[t]{2}{*}{ me..$^{-1}$} & Húmedo & 3,88 & 3,98 & 0,58 & 1,29 & 1,29 & 4,63 \\
\hline & & Seco & 7,54 & 3,15 & 1,40 & 1,63 & 1,42 & 2,00 \\
\hline \multirow[t]{2}{*}{ Cloruro } & \multirow[t]{2}{*}{ me..$^{-1}$} & Húmedo & 8,90 & 12,20 & 1,94 & 3,38 & 3,49 & 34,87 \\
\hline & & Seco & 15,10 & 16,45 & 4,17 & 4,73 & 4,17 & 9,30 \\
\hline
\end{tabular}

Fuente: Elaboración propia (2016).

\section{Indicadores de aptitud de las aguas para el riego}

Los criterios analizados en el punto anterior restringen el uso de estas aguas para el riego, sin embargo, se hizo un análisis más detallado con el empleo de otros indicadores y criterios más específicos para ver cuán perjudicial puede ser el uso agrícola de este recurso, los resultados se muestran en la Tabla III. 
Yoandris Garcia-Hidalgo. Index of vulnerability of water resources for irrigation in the basin of Naranjo river, Cuba

Tabla III. Indicadores de la aptitud de las aguas para utilizarlas en el riego.

\begin{tabular}{|c|c|c|c|c|c|c|c|c|}
\hline \multirow{2}{*}{ Variables } & \multirow{2}{*}{ Unidades } & \multirow{2}{*}{ Período } & \multicolumn{6}{|c|}{ Muestras } \\
\hline & & & I & II & III & IV & V & VI \\
\hline \multirow{2}{*}{$\begin{array}{l}\text { Salinidad } \\
\text { Efectiva }\end{array}$} & \multirow[t]{2}{*}{ me..$^{-1}$} & Húmedo & 12,38 & 12,43 & 5,30 & 7,17 & 7,17 & 9,50 \\
\hline & & Seco & 8,83 & 8,46 & 3,49 & 3,87 & 3,32 & 6,82 \\
\hline \multirow{2}{*}{$\begin{array}{l}\text { Salinidad } \\
\text { Potencial }\end{array}$} & \multirow[t]{2}{*}{ me..$^{-1}$} & Húmedo & 10,84 & 14,19 & 2,23 & 4,03 & 4,14 & 37,19 \\
\hline & & \begin{tabular}{|l} 
Seco \\
\end{tabular} & 18,87 & 18,02 & 4,87 & 5,54 & 4,88 & 10,30 \\
\hline \multirow[t]{2}{*}{ RASx } & \multirow[t]{2}{*}{$\mathrm{U}$} & Húmedo & 8,2 & 8,4 & 4,4 & 4,4 & 4,4 & 2,7 \\
\hline & & \begin{tabular}{|l|} 
Seco \\
\end{tabular} & 19,7 & 5,4 & 9,6 & 7,3 & 11,5 & 4,4 \\
\hline \multirow{2}{*}{$\begin{array}{l}\text { Porciento de } \\
\text { Sodio Posible }\end{array}$} & \multirow[t]{2}{*}{$\%$} & Húmedo & 146 & 150 & 83 & 75 & 75 & 107 \\
\hline & & \begin{tabular}{|l} 
Seco \\
\end{tabular} & 79 & 45 & 75 & 69 & 78 & 47 \\
\hline \multirow{2}{*}{$\begin{array}{l}\text { Porciento de } \\
\text { Sodio Soluble }\end{array}$} & \multirow[t]{2}{*}{$\%$} & Húmedo & 56 & 57 & 62 & 61 & 61 & 18 \\
\hline & & Seco & 79 & 45 & 75 & 69 & 78 & 47 \\
\hline \multirow{2}{*}{$\begin{array}{l}\text { Índice de } \\
\text { Langellier }\end{array}$} & \multirow[t]{2}{*}{$\mathrm{U}$} & Húmedo & 1,1 & 1,1 & 0,4 & 0,6 & 0,6 & 0,9 \\
\hline & & \begin{tabular}{|l|} 
Seco \\
\end{tabular} & 1,5 & 1,6 & 1,1 & 1,0 & 0,9 & 1,0 \\
\hline
\end{tabular}

Fuente: Elaboración propia (2016).

\section{Salinidad}

Los valores de SST y RAS son incluidos en el diagrama de Wilcox para clasificar las aguas con fines de riego. En la Figura II se puede observar que las fuentes superficiales son altamente salinas (C3) y las subterráneas son extremadamente salinas (C4), por tanto el peligro de salinización de los suelos al regar con estas aguas es alto o muy alto. En cuanto al sodio, las fuentes superficiales en el período húmedo tienen baja sodicidad (S1), en el resto la sodicidad es media (S2) e incluso hay un caso de muy alta sodicidad (S4). Esto indica que los peligros de alcalinización de los suelos provocados por el sodio son medios. 
Figura II. Clasificación de las aguas de riego de la cuenca del río Narango según el diagrama de Wilcox.

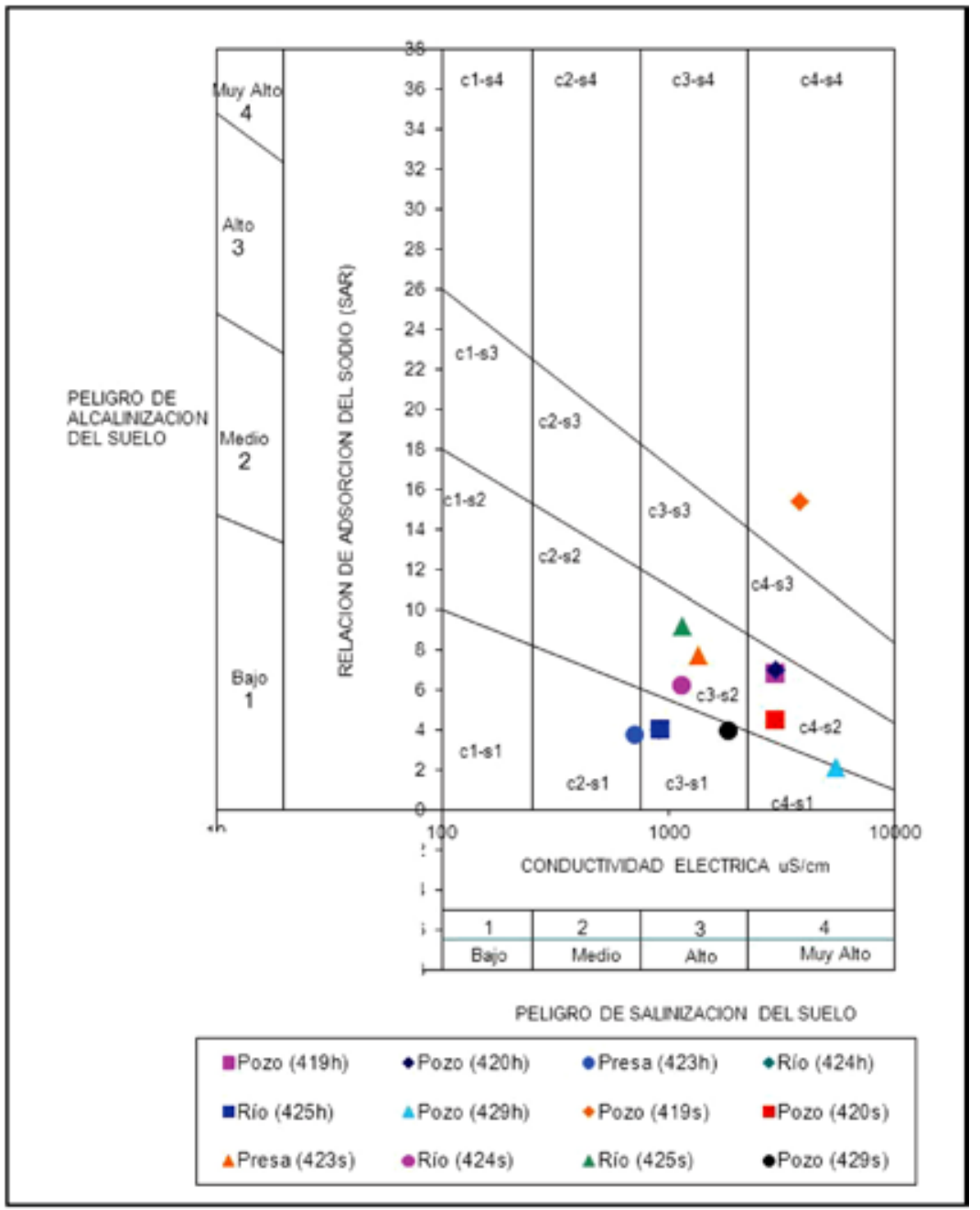

Según la Salinidad efectiva (SE) estas aguas se clasifican como Condicionadas para su empleo en el riego $\left(3-10 \mathrm{me}^{-\mathrm{l}^{-1}}\right)$, ya que pueden pasar a formar parte del agua del suelo en forma de sales menos solubles como carbonato de calcio y magnesio o sulfato de calcio.

Por el criterio de Salinidad potencial (SP), que mide el peligro de los cloruros y sulfatos al actuar a bajos niveles de humedad y aumentar 
la presión osmótica, las fuentes subterráneas son No recomendables ( $>15$ me. $\left.1^{-1}\right)$, mientras las superficiales están Condicionadas para el riego.

\section{Efectos sobre la infiltración}

Según el Porciento de sodio posible (PSP) las aguas están Condicionadas, pues en todos los casos el valor es superior al $50 \%$, existiendo peligro de sustitución del calcio y el magnesio del complejo de cambio por el sodio.

El Porciento de sodio soluble (PSS) expresa la proporción de sodio y potasio respecto al total de cationes adsorbidos. Si ese porcentaje es muy alto el sodio puede alcanzar concentraciones elevadas en el complejo de cambio y provocar el deterioro físico de los suelos. Las aguas de fuentes superficiales son clasificadas como Dudosas $(60-80 \%)$, mientras las subterráneas son Permisibles $(40-60 \%)$.

\section{Toxicidad}

La magnitud de la toxicidad por la presencia de cloruros en las aguas es valorada en función del método de riego. Debido a que en las técnicas de riego por gravedad prácticamente no hay contacto del agua con zonas de transpiración más intensa (bordes y puntas de las hojas) los tenores asimilados son mayores, aun así las restricciones de uso son severas para las fuentes subterráneas y ligeras a moderadas para las superficiales. Si el riego es por aspersión en riesgo es mayor (severo) pues el agua se deposita en las hojas y puede provocar necrosis.

La toxicidad por sodio tiene un comportamiento similar a los cloruros. Los valores de RASx son en todos los casos superiores a 3,0 me. $\mathrm{l}^{-1}$, a partir del cual se consideran ligeras a moderadas, si son superiores a 9,0 me. $1^{-1}$ pasan a la categoría de severas.

El riesgo de obstrucción de los sistemas de riego presurizados es alto $(0,5-1,0)$ o muy alto $(>1,0)$ según el Índice de Langellier. Este parámetro valora la posibilidad de precipitación del calcio en forma de carbonato cuando alcanza la saturación en presencia de bicarbonato (Fuentes, 2003) [9]. 


\section{Conclusiones}

La vulnerabilidad del recurso hídrico en relación a la oferta hídrica, estrés hídrico, reporta reducciones en la cuenca; se detecta el incremento, en algunas estaciones para el período evaluado, la cuenca del río Naranjo está sometida a stress hídrico basado en el índice de disponibilidad per cápita.

Los indicadores de calidad del agua evaluados arrojaron alta conductividad eléctrica que las hace extremadamente salinas, son no aptas para el riego.

La vulnerabilidad es alta, debido al muy bajo índice de retención y regulación del recurso hídrico y las presiones moderadas de uso con respecto a la oferta hídrica superficial disponible.

La vulnerabilidad futura ante el cambio climático puede reducirse si se toman acciones que resulten en mejoras de la infraestructura, los servicios y la condición de las personas. Algunas acciones que pueden colaborar en reducir la vulnerabilidad incluyen una mayor aplicación del conocimiento y conciencia acerca de las interacciones entre el clima y la sociedad; mejor tecnología y herramientas para la planificación, educación y salud, y prevención del riesgo. Si el país logra además dirigir recursos hacia aquellos cantones con mayor índice de vulnerabilidad presente y futura, el país podrá reducir su vulnerabilidad ante los efectos del cambio climático en el sector de recursos hídricos.

\section{Referencias}

Ayers, R. S. Westcot, D.W. (1987). La calidad del agua en la agricultura. Roma: Estudio FAO. Revista Riego y Drenaje, 29(1) 35 págs.

Carreño, M. (2013). Sistemas de indicadores para la evaluación de riesgos. Costa Rica- 115 págs.

Chaves, H., y Alipaz, S. (2007). An Integrated Indicator Based on Basin Hydrology, Environment, Life, and Policy: The Watershed Sustainability Index. Water Resources Management.; (2007). 21:883-895.

CONAGUA. (2015), Vulnerabilidad de los recursos hídricos de México ante el cambio climático.

Fuentes, J. L. (2003). Técnicas de riego. 4a edición revisada y ampliada ed. Madrid: Coedición Ministerio de Agricultura, Pesca y Alimentación - Ediciones Mundi-Prensa, 483 p. 
IDEAM (2010). Propuesta de indicadores del régimen hidrológico Instituto de Hidrología, Meteorología y Estudios Ambientales. Ministerio de Medio Ambiente, Vivienda y Desarrollo territorial. Bogotá, Colombia. 2010.

IPCC. (2007). Cambio Climático 2007: Informe de Síntesis. Ginebra, Suiza: Informe del Grupo Intergubernamental de Expertos sobre el cambio climático.

IPCC. 2008.. El Cambio Climático y el agua. Ginebra, Suiza.

IPPC. (2007). Climate Change: impacts, adaptation and vulnerability. Contribution of Working Group II to the Fourth Assessment Report of the Intergovernmental Panel on Climate Change. Cambridge, UK: Cambridge University Press

Magrin, et. al. Peña, Fernández (2007). Latina America, Climate Change 2007: Impacts, Adaptation and Vulnerability, Contribution of Working Group II to the Fourth Assessment Report of the Intergovernmental Panel on Climate Change. Cambridge University Press.

Rosenzweig, et.al. Ramirez, Perdomo (2004). Water Resources for agriculture in a changing climate: International case studies. Global Environmental Change, 14(4) 135 págs.

Sharma, H (2009). Application of climate information and predictions in water sector: capabilities. WCC-3. Geneve.

Troballes, J (2015).Troballes.org/wp-content/uploads/Agua-un-elementoesencial-para-la-vida.pdf 